\title{
Surface Impedance Characterization for Mutual Coupling Calculation of Patches on a Dielectric-Coated PEC Circular Cylinder
}

\author{
Andrés García-Aguilar \\ Signals, Systems and \\ Radiocommunications Department; \\ Technical University of Madrid. \\ Avda. Complutense, 30, \\ 28040 Madrid, Spain. \\ andresg@gr.ssr.upm.es
}

\author{
Zvonimir Sipus \\ Faculty of Electrical \\ Engineering and Computing; \\ University of Zagreb. \\ Unska 3, \\ 10000 Zagreb, Croatia. \\ zvonimir.sipus@fer.hr
}

\author{
Manuel Sierra-Pérez \\ Signals, Systems and \\ Radiocommunications Department; \\ Technical University of Madrid. \\ Avda. Complutense, 30, \\ 28040 Madrid, Spain. \\ m.sierra.perez@gr.ssr.upm.es
}

\begin{abstract}
A novel formulation for the surface impedance characterization is introduced for the canonical problem of surface fields on a perfect electric conductor (PEC) circular cylinder with a dielectric coating due to a electric current source using the Uniform Theory of Diffraction (UTD) with an Impedance Boundary Condition (IBC). The approach is based on a TE/TM assumption of the surface fields from the original problem. Where this surface impedance fails, an optimization is performed to minimize the error in the SD Green's function between the original problem and the equivalent one with the IBC. This asymptotic method, accurate for large separations between source and observer points, in combination with spectral domain (SD) Green's functions for multidielectric coatings leads to a new hybrid SD-UTD with IBC to calculate mutual coupling among microstrip patches on a multilayer dielectric-coated PEC circular cylinder. Results are compared with the eigenfunction solution in SD, where a very good agreement is met.
\end{abstract}

\section{INTRODUCTION}

Recently, a new Uniform Theory of Diffraction (UTD) based asymptotic solution with impedance boundary conditions (IBC) has been introduced for surface field determination on a circular cylinder [1]. UTD with IBC Green's functions combined with Method of Moments (MoM) are able to obtain the mutual coupling between patches. Nevertheless, although some progress has been done to calculate efficiently proper Green's functions [2], very few attention has been paid about how to figure out surface impedance for each specific case, because it must change with the inclination angle of the geodesic rays along the structure.

In this paper, the surface impedance is derived in the SD by performing a TE/TM decomposition of the surface fields. UTD based asymptotic Green's functions with IBC are slightly modified to include the surface impedance dependence with the geometrical parameters of the rays upon the cylinder. Since UTD with IBC solution would be valid only for one dielectric layer and for a large separation between the source and the observation point, it is proposed its hybridization with a SD approach [3], thus extending the method to multilayer structures and increasing the accuracy of the surface fields calculated. Here, nonparaxial region has only been considered.

\section{Method OF AnALYSis}

The geometry of the problem is a perfectly conducting patch located over the surface of a multilayer dielectric-coated PEC circular cylinder. It is assumed that the cylinder is infinite in the axial direction. The Electric Field Integral Equation (EFIE) solved by MoM can be accelerated combining an asymptotic UTD based solution of the surface fields, as it was proposed in [4] for aperture conformal array antennas. The hybrid method consists into a extraction procedure of the Green's function in the MoM matrix element, as

$$
\begin{aligned}
& Z_{j i}= \frac{1}{4 \pi^{2}} \sum_{m=-\infty}^{\infty} \int_{-\infty}^{\infty} \underline{\tilde{W}}_{j}\left(-m,-k_{z}\right) \\
& \cdot\left(\underline{\underline{G}}^{e, J}\left(m, k_{z}\right)-\underline{\underline{G}}_{a s y m}^{e, J}\left(m, k_{z}\right)-\right) \cdot \underline{\tilde{J}}_{i}\left(m, k_{z}\right) d k_{z} \\
& \\
&+\int_{S} \int_{S^{\prime}} \underline{W}_{j}(\phi, z) \cdot \underline{\underline{G}}_{a s y m}^{e, J}\left(d, \phi-\phi^{\prime}, z-z^{\prime}\right) \\
& \cdot \underline{J}_{i}\left(\phi^{\prime}, z^{\prime}\right) d S d S^{\prime}
\end{aligned}
$$

where $\underline{\underline{G}}_{\text {asym }}^{e, J}$ is the asymptotic UTD based Green's function.

To simplify the computation of this asymptotic part, the boundary conditions on a dielectric coated PEC surface can be approximated by an IBC [1]. In this work, two different surface impedances have been used depending on the electric and magnetic field ratios. This method consists into assume a TE/TM decomposition of the fields propagating along the cylinder. The IBC suggests that this approximation is acceptable. TE/TM surface impedance, from the eigenfunction solution, is expressed in terms of Bessel functions of first and second kind as 


$$
\begin{aligned}
& \tilde{Z}_{s e}=-\frac{j k_{0} Z_{c 0}}{k_{\rho 1}} \cdot \frac{J_{m}^{\prime}\left(k_{\rho 1} d\right) Y_{m}^{\prime}\left(k_{\rho 1} a\right)-J_{m}^{\prime}\left(k_{\rho 1} a\right) Y_{m}^{\prime}\left(k_{\rho 1} d\right)}{J_{m}\left(k_{\rho 1} d\right) Y_{m}^{\prime}\left(k_{\rho 1} a\right)-J_{m}^{\prime}\left(k_{\rho 1} a\right) Y_{m}\left(k_{\rho 1} d\right)} \\
& \tilde{Z}_{s m}=\frac{j k_{\rho 1} Z_{c 0}}{\varepsilon_{r 1} k_{0}} \cdot \frac{J_{m}\left(k_{\rho 1} d\right) Y_{m}\left(k_{\rho 1} a\right)-J_{m}\left(k_{\rho 1} a\right) Y_{m}\left(k_{\rho 1} d\right)}{J_{m}^{\prime}\left(k_{\rho 1} d\right) Y_{m}\left(k_{\rho 1} a\right)-J_{m}\left(k_{\rho 1} a\right) Y_{m}^{\prime}\left(k_{\rho 1} d\right)}
\end{aligned}
$$

where subscript 0 denotes the upper dielectric slab and 1 the lower dielectric layer. This TE/TM surface impedance is shown to be valid for low $\alpha$ angles and only for for $z z^{\prime}$ and $\phi \phi^{\prime}$ field and source orientation. It is possible to make an optimization of the TE/TM surface impedance for the cases where they fail by shifting the root of the TE surface impedance and tracking its slope to minimize the average relative error between the amplitudes of the SD Green's function for the original problem and for the equivalent problem.

Furthermore, to increase the computation efficiency in the surface impedance formulas when calculating Fock-type integrals, they can be approximated with a ratio of two $n t h$-order polynomials as a function of the spectral variable $k_{t}=m / d$.

\section{RESUlts AND CONCLUSIONS}

To validate the TE/TM surface impedance hypothesis some results have been performed. Fig. 1 shows a comparison of the SD Green's functions in amplitude and phase between the eigenfunction solution and the IBC with a second-order polynomial interpolation and without it. Surface impedance optimization is needed for angles larger than $25^{\circ}$ for $z z^{\prime}$ and $\phi \phi^{\prime}$ cases and for all $\alpha$ angles for $z \phi^{\prime}$ component. A very good agreement is met in SD, even for $\phi \phi^{\prime}$ where nonoptimized TE/TM surface impedance approach is not valid because couplings between modes are very strong.

The mutual impedance between two sinusoidal electric current modes for two small dipoles, by taking the second term of equation (1), is shown in Fig. 2, whose values are comparable with results in reference [5]. For these geometry, surface impedance optimization is not necessary, and a fourth order polynomial interpolation has been used.

\section{ACKNOWLEDGMENT}

The project has the support of the Spanish Education Ministry under reference TEC2008-06736/TEC and TEC201128789-C02-01, and a Spanish Government Research Fellowship (FPI) under reference BES-2009-021462.

\section{REFERENCES}

[1] Ç. Tokgöz and R. J. Marhefka, "A UTD based asymptotic solution for the surface magnetic field on a source excited circular cylinder with an impedance boundary condition," IEEE Transactions on Antennas and Propagation, vol. 54, no. 6, pp. 1750-1757, Jun. 2006.

[2] B. Alisan, V. B. Ertürk, and A. Altintas, "Efficient computation of nonparaxial surface fields excited on a electrically large circular cylinder with an impedance boundary conditions," IEEE Transactions on Antennas and Propagation, vol. 54, no. 9, pp. 2559-2567, Sep. 2006.

[3] Z. Sipus, P.-S. Kildal, R. Leijon, and M. Johansson, "An algorithm for calculating Green's function of planar, cylindrical and spherical multilayer substrates," ACES J., vol. 13, no. 3, pp. 243-254, Nov. 1998.

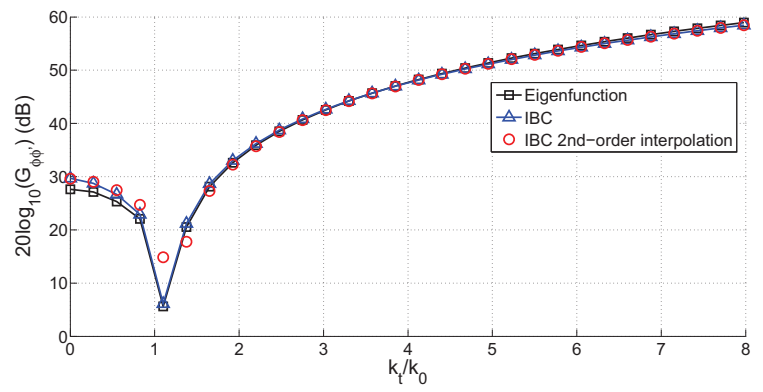

(a) Amplitude.

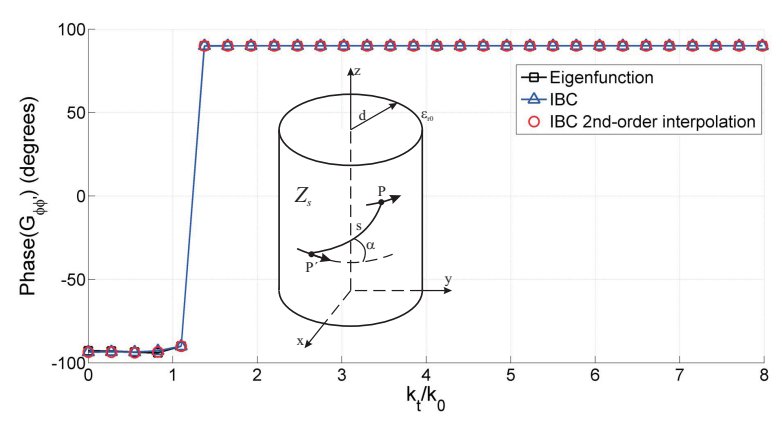

(b) Phase.

Fig. 1. SD Green's function for $\phi \phi^{\prime}$ component, with $f=4 G H z, a=3 \lambda_{0}$, $\varepsilon_{r 1}=2.2, \varepsilon_{r 0}=1, t=0.762 \mathrm{~mm}$ and $\alpha=40^{\circ}$.

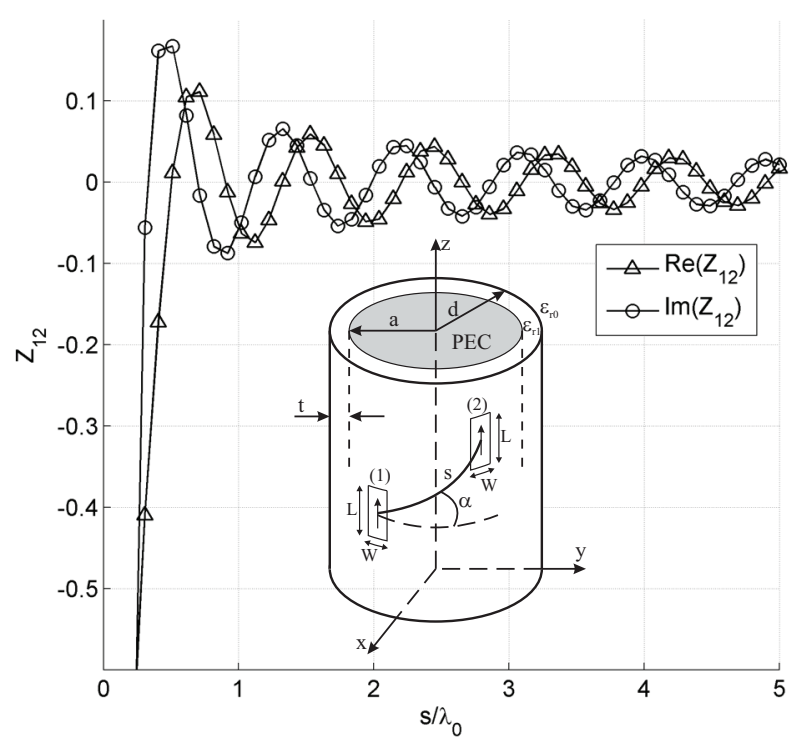

Fig. 2. Mutual impedance for $z z^{\prime}$ component, with $a=3 \lambda_{0}, \varepsilon_{r 1}=3.25$, $\varepsilon_{r 0}=1, t=0.06 \lambda_{0}, W=0.02 \lambda_{0}, L=0.05 \lambda_{0}$ and $\alpha=55^{\circ}$.

[4] M. Bosiljevac, P. Persson, and Z. Sipus, "Efficient analysis of aperture antennas on generally shaped convex multilayered surfaces using a hybrid SD-UTD method," IEEE Transactions on Antennas and Propagation, vol. 57, no. 5, pp. 1420-1431, May 2009.

[5] V. B. Ertürk and R. G. Rojas, "Efficient computation of surface fields excited on a dielectric-coated circular cylinder," IEEE Transactions on Antennas and Propagation, vol. 48, no. 10, pp. 1507-1516, Oct. 2000. 